\title{
Physical Limits on Atomic Resolution
}

\author{
D. Van Dyck, ${ }^{1, \star}$ S. Van Aert, ${ }^{2}$ and A.J. den Dekker ${ }^{2}$ \\ ${ }^{1}$ Department of Physics, University of Antwerp, 2020 Antwerp, Belgium \\ ${ }^{2}$ Delft Center for Systems and Control, Delft University of Technology, 2628 CD Delft, The Netherlands
}

\begin{abstract}
It is shown that the ultimate resolution is not limited by the bandwidth of the microscope but by the bandwidth (i.e., the scattering power) of the object. In the case of a crystal oriented along a zone axis, the scattering is enhanced by the channeling of the electrons. However, if the object is aperiodic along the beam direction, the bandwidth is much more reduced. A particular challenge are the amorphous objects. For amorphous materials, the natural bandwidth is that of the single atom and of the order of $1 \AA^{-1}$, which can be reached with the present generation of medium voltage microscopes without aberration correctors. A clear distinction is made between resolving a structure and refining, that is, between resolution and precision. In the case of an amorphous structure, the natural bandwidth also puts a limit on the number of atom coordinates that can be refined quantitatively. As a consequence, amorphous structures cannot be determined from one projection, but only by using atomic resolution tomography. Finally a theory of experiment design is presented that can be used to predict the optimal experimental setting or the best instrumental improvement. Using this approach it is suggested that the study of amorphous objects should be done at low accelerating voltage with correction of both spherical and chromatic aberration.
\end{abstract}

Key words: transmission electron microscope, amorphous structure, atomic resolution, precision parameter estimation, electron tomography

\section{INTRODUCTION}

Recently there have been exciting new developments in the correction of electron microscopic aberrations that will give a boost to the high-resolution electron microscopy (HREM) field but that may also generate unrealistic expectations. One could, for instance, believe that if the microscope becomes perfect one can resolve amorphous structures. This is a wrong conception. Indeed, in the image formation process in the electron microscope, several band-limiting factors cause a loss in resolution, some of which can be eliminated by instrumental improvements, such as the coherent and incoherent aberrations of the microscope, the modulation transfer junction (MTF) of the camera, and so forth. The ultimate limit on the resolution, however, cannot be eliminated by physical means. Because the electron interacts with the electrostatic potential of the atom, the "width" of the atom potential itself, even blurred by thermal vibrations, poses the ultimate limit on the resolution. For instance, the "width" of a Si atom is of the order of $1 \AA$. In the case of a crystal viewed along a zone axis, the situation is different. In that case, the atoms along the column act as lenses so as to focus the electron beam at regular depths.

Received January 6, 2003; accepted June 6, 2003.

${ }^{\star}$ Corresponding author. E-mail: dirk.vandyck@ua.ac.be
Hence, at an appropriate thickness, the exit wave is more sharply peaked than the atom potential itself and the resolution is increased. In that situation the ultimate resolution is given by the width of the $1 s$ state of the column.

In the case of an amorphous object, however, the atom cores do not superimpose along the beam direction and the bandwidth is determined by the scattering factor of the single atom. It is known, for instance, that it is impossible to determine the resolution of a modern EM using amorphous Si because the amount of scattered information beyond $1 \AA$ is insufficient.

The study of amorphous objects can thus be considered as the ultimate challenge for HREM. In this work, we focus on the problem of the quantitative determination of an amorphous structure and the physical limits.

First, we have to make a distinction between resolving a structure and quantitative refining, or between resolution and precision.

\section{Classical Resolution}

The classical definition of resolution, which goes back to Lord Rayleigh, is related to the point-spread function of the imaging instrument irrespective of the noise due to the counting statistics. We will introduce counting statistics later on. For simplicity, we will assume this point-spread 
function to be a two-dimensional Gaussian function of the following form:

$$
p(r)=\frac{1}{2 \pi \rho^{2}} \exp \left(-\frac{r^{2}}{2 \rho^{2}}\right)
$$

where $r$ is the absolute value of the two-dimensional vector $r$ and $\rho$ is the "width" of the Gaussian function. According to Rayleigh, the point resolution $\rho_{p}$, that is, the smallest distance at which two points can be resolved, is given by the requirement that the value of the cross section of the composite intensity distribution halfway between these two points is about 0.8 times the value at the maxima. Thus,

$$
2 \exp \left(\frac{-\rho_{p}^{2}}{8 \rho^{2}}\right)=0.8
$$

from which it follows that

$$
\rho_{p} \approx 2 \sqrt{2} \rho
$$

It corresponds in a sense to the "width" of the point-spread function of the imaging instrument. In electron microscopy, this includes the point spread due to the lens aberrations, the incoherent effects, the vibrations and stray fields, and the detector. Moreover, it has to be taken into account that the atoms, including their thermal vibrations, are no point scatterers and, hence, have their own bandwidth. Each effect contributing to the imaging process can be represented by a transfer function, which acts as a low-pass filter. The transfer function of the electron microscope consists of a damping function, which is mainly due to chromatic aberration, and a phase shift, which causes the oscillations. Because there are different ways to get rid of the oscillations, such as focal series reconstruction and correction of the spherical aberration, the Rayleigh resolution of the electron microscope can be assumed to be given by the so-called information limit, which is proportional to the inverse of the highest spatial frequency that is still transferred with appreciable intensity. For simplicity, it will be assumed that the imaging process is linear. This requires that the interaction between the electron and the object is linear. If the object is a crystal, viewed along a zone axis, the electrostatic potential of all the atoms along the atom column superimpose, which makes the interaction very strong and highly nonlinear, so that this approximation does not apply. In that particular case, due to the focusing effect of the successive atoms, the scattering is increased to much higher angles. This effect is explained by the channeling theory (Van Dyck \& Op De Beeck, 1996). However, for amorphous objects, the atoms are stacked in a disordered fashion, so that, in projection, their cores do not overlap, except by coincidence. As a result, the interaction remains linear for much larger object thicknesses. If the imaging is linear, all transfer functions have to be multiplied, or, equivalently, the point-spread functions have to be convolved. If it is assumed that all constituent point spread functions are Gaussian, the resulting function is a Gaussian as well, with a Rayleigh resolution $\rho_{p}$ determined by

$$
\rho_{p}^{2}=\rho_{A}^{2}+\rho_{T}^{2}+\rho_{E M}^{2}+\rho_{v}^{2}+\rho_{D}^{2},
$$

with $\rho_{A}$ the "width" of the electrostatic potential of the atom, representing the scattering of the atom, $\rho_{T}$ the Rayleigh resolution limited by thermal vibrations of the atom, $\rho_{E M}$ the Rayleigh resolution of the electron microscope, $\rho_{v}$ the Rayleigh resolution limited by the environment (vibrations and stray fields), and $\rho_{D}$ the Rayleigh resolution limited by the detector. Today, for the best electron microscopes, $\rho_{E M}$ is somewhat below $1 \AA$. By future instrumental developments one can improve this resolution by improving the resolutions of all different subchannels. However, a factor that one cannot improve is the resolution limit of the atom itself, $\rho_{A}$. At this point it is already difficult to find suitable objects that can be used to demonstrate the true resolution $\rho_{E M}$ of an electron microscope. For amorphous silicon, $\rho_{A}$ is about $1 \AA$. Therefore, if amorphous silicon is observed with an ideal electron microscope, the total resolution is $\rho_{p} \approx 1 \AA$.

For quantitative structure determination, this classical concept is not appropriate and we will have to follow a model-based approach.

\section{Resolution for Counting Statistics}

Another resolution criterion was proposed by Rose (1948). It is based on the following arguments. Suppose the image detector system is able to detect the individual particles (electrons) from the image. Let us call $D$ the dose, that is, the number of electrons per unit area. Consider now a square of size $\rho \times \rho$. The total number of particles in the square is then

$$
N=D \rho^{2}
$$

From Poisson statistics, the standard deviation (error bar) is $\sqrt{N}$ and the signal-to-noise (SNR) ratio is then

$$
S N R=\frac{N}{\sqrt{N}}=\sqrt{N}=\rho \sqrt{D} .
$$

The result can be interpreted as follows. $\rho$ is the smallest meaningful unit of resolution given an imaging dose $D$ and a desired SNR. 


\section{Deterministic Model-Based Resolution}

The concepts of classical resolution and resolution from counting statistics are both special cases of a more general concept.

The classical definition of point resolution according to Rayleigh expresses the fact that if one has no prior knowledge about the object and if the image is interpreted visually (qualitatively), the smallest observable detail is determined by the size of the "blurring" of the instrument. However, the situation changes completely if one has a model for the object and if the image contrast can be measured quantitatively (den Dekker \& van den Bos, 1997), because the atoms constitute the "alphabet" of matter. We dispose of a unique opportunity for a model-based approach to interpret the images, provided the microscope would have sufficient resolution to visualize the individual atoms.

Because all the imaging steps in the electron microscope are known, one can compute the image of an atom. Thus, there is no interest in the detailed form of this image, but only in the position of the atom. The only objective of the experiment is to determine this position. Obviously, in the absence of noise, numerically fitting the known onepeak model to the image with respect to the position parameter would result in a perfect fit. The resulting solution for this location would be exact, and despite blurring, no limit to location resolution would exist.

This line of reasoning can be extended to position measurements of atoms in amorphous structures from noise-free electron microscopic observations. In a sense, one is looking for the optimum value of a criterion in a parameter space whose dimension is equal to the number of parameters to be measured, which is equal to $2 n$, if $n$ is the total number of atoms. The factor 2 accounts for the $x$ - and $y$-coordinates of the projected position. Each possible combination of the $2 n$ parameters can be represented by a point in a $2 n$-dimensional space. The search for the global optimum of the criterion of goodness of fit in this space is an iterative numerical optimization procedure. However, the problem with which one can be faced is of computational kind. The existing optimization methods fail if the dimension of the parameter space is so high that one cannot avoid ending up at a local optimum instead of at the global optimum of the criterion of goodness of fit, so that the wrong structure is suggested. To solve this dimensionality problem, that is, to find a pathway to the global optimum, a good starting structure is required, that is, initial conditions should be available for the parameters. In other words, the structure has to be resolved.

This procedure is comparable to X-ray crystallography where first one tries to "resolve" the atomic structure of a crystal using so-called "direct methods" and afterward one tries to "refine" the structure iteratively.

If the atoms are resolvable from the image, or, in other words, if the individual atoms can be visualized, one can use the positions of the observed maxima in the image as starting positions for the atoms.

A big problem arises if the interatomic distances are smaller than the resolution. Then, the images of most neighboring atoms overlap so that the individual atoms are not resolved. In that case, one cannot find a reasonable trial structure to start the refinement. Due to the strong overlap of the neighboring atoms, the influence of all the coordinates is, in a sense, coupled in the image. One cannot alter one coordinate without affecting the others. Finding the optimal fit then becomes an optimization problem with many strongly correlated parameters. This introduces severe problems of the computational kind. Computational problems can only be overcome if the individual atoms in projection are resolved, which puts a severe restriction on the maximal thickness of amorphous objects.

For the amorphous object, the number of parameters increases with thickness. Therefore, from a certain thickness on, it will be difficult to resolve the structure. To resolve the structure, it will be assumed that the distances between neighboring projected atom positions should be larger than or equal to the Sparrow resolution $\rho_{s}$.

Compared to Rayleigh resolution, which is based on presumed capabilities of the human visual system, the Sparrow resolution is based on a less subjective criterion, which is valid for a hypothetical perfect imaging instrument. It states that the smallest resolvable distance between two points is that for which the minimum in the composite image intensity distribution just disappears (den Dekker \& van den Bos, 1997). The Sparrow resolution is given in terms of the Raleigh resolution by

$$
\rho_{s} \approx \frac{\sqrt{2} \rho_{p}}{2}
$$

The reason for choosing this criterion is that the computer will then be able to distinguish the individual atoms, because the observations are assumed to be noise free. However, it should be noticed that this criterion is not exact, and, therefore, it will only give rough guidelines. Suppose that the mean concentration of atoms per cubic angstrom is equal to $V$. Then, the mean concentration $A$ of projected atoms per square angstrom is given by

$$
A=V z
$$

where $z$ is the thickness of the amorphous foil. On the other hand, if it is assumed that each projected atom occupies a circle with a diameter equal to the average distance $d$, averaged over distances between nearest neighbor projected atoms, then,

$$
A \approx \frac{1}{\pi\left(\frac{d}{2}\right)^{2}} .
$$


From this it follows that the thickness of the amorphous foil is approximately given by

$$
z \approx \frac{4}{\pi d^{2} V}
$$

To resolve the structure and therefore to avoid dimensionality problems, it will be assumed that the following condition is met:

$$
d \geq 2 \rho_{s} .
$$

Then, it follows from equations (7) and (8), that

$$
z \leq \frac{1}{\pi \rho_{s}^{2} V}
$$

For amorphous silicon, it follows from equation (5) that $\rho_{s}$ is approximately equal to $0.7 \AA$. Furthermore, for amorphous silicon, $V$ is approximately equal to 0.05 atoms per cubic angstrom. Hence, it follows from equation (10) that the amorphous silicon foil should not exceed thicknesses of the order of $13 \AA$ so as to avoid dimensionality problems. This thickness is rather small, which means that it is unrealistic to expect that atomic resolution TEM is able to resolve amorphous silicon samples with realistic foil thicknesses from only one projection.

\section{TOMOGRAPHY}

To resolve and refine amorphous objects one has to use electron tomography. In this technique, the object is tilted in incremental angles and for each orientation a projection is taken.

The Fourier transform of a projection yields a section through the origin of the three-dimensional Fourier space. By combining many different projections, one can then reconstruct the whole Fourier space. The number of images (data planes) is much larger, whereas the number of atom coordinates increases only from 2 to 3 . In that case, it can be shown that a microscope resolution of $0.2 \mathrm{~nm}$ is sufficient to resolve and refine amorphous structures.

\section{Precision}

Thus far it has been assumed that the observations are noise free. However, in any real-life experiment, the observations are prone to noise.

Suppose that one has a CCD camera that is able to count the individual photons forming the image of a single atom. The noise on these images stems from the counting statistics. The position parameters can be estimated by numerically fitting the known parameterized mathematical model to the images with respect to the component positions.

In practice, one starts from a trial structure for the atom positions and one simulates the image. From the mismatch between experimental and simulated images, one can correct for the atom positions. This is an iterative procedure. At each iteration, the fit improves until finally convergence is reached. If the atoms are resolvable from the beginning, the iteration only alters the atom positions slightly so that convergence to the optimal solution is guaranteed. The precision, that is, the standard deviation, on the atom positions that can thus be reached can be adequately quantified in the form of a lower bound $s_{L B}$ on the standard deviation (van den Bos \& den Dekker, 2001).

Applying statistical parameter estimation theory, the attainable precision can be adequately quantified in the form of the so-called Cramér-Rao lower bound (CRLB; den Dekker \& van den Bos, 1997). This is a lower bound on the variance of any unbiased estimator of a parameter. It means that the variance of different estimators, such as the least squares or the maximum likelihood estimator, can never be lower than the theoretical CRLB on the variance.

First, the position measurement of one isolated point object will be investigated. Let us demonstrate this for the simple case where the point-spread function is assumed to be Gaussian and if the total number of imaging particles is $N$, the lower bound on the standard deviation $s_{L B}$ of the coordinates estimates of the position, that is, the square root of the CRLB, is given by

$$
s_{L B} \approx \frac{\rho}{\sqrt{N}},
$$

or, from equation (3)

$$
s_{L B} \approx \frac{\rho_{p}}{2 \sqrt{2 N}} .
$$

Thus, the precision with which the position can be determined is a function of both the Rayleigh resolution $\rho_{p}$ and the number of imaging photons $N$. If $N$ is large, the precision can be orders of magnitude higher than the point resolution $\rho_{p}$.

It is an interesting exercise to see what effect aberration correctors and monochromators might have on the precision. In Figure 1, the lower bound $s$ on the standard deviation of the position of a single projected atom is evaluated as a function of $C_{s}$ for a $300-\mathrm{keV}$ electron microscope. This is done for three possible cases: The first case is a microscope without monochromator and without $C_{c}$ corrector (energy spread, expressed as full width at half maximum height, of $1.7 \mathrm{eV}$ and $C_{c}$ of $1.3 \mathrm{~mm}$ ), the second case is a microscope with monochromator (energy spread of $0.2 \mathrm{eV}$ and $C_{c}$ of $1.3 \mathrm{~mm}$ ), and the third case is a microscope with $C_{c}$ corrector (energy spread of $1.7 \mathrm{eV}$ and $C_{c}$ of $0 \mathrm{~mm}$ ). 


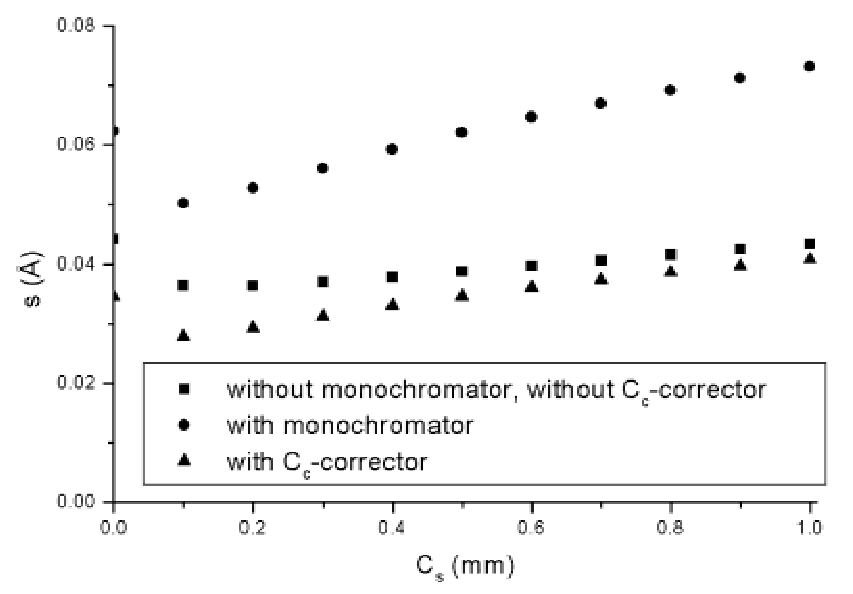

Figure 1. Lower bound $s$ on standard deviation of a single Si atom position as a function of $C_{s}$ for a microscope with an accelerating voltage of $300 \mathrm{keV}$. Three possible cases are distinguished: without monochromator and without $C_{c}$ corrector, with monochromator, and with $C_{c}$ corrector. At each setting, the recording time is kept constant.

At each setting, the recording time is kept constant, assuming that specimen drift puts a practical limit to the experiment. As a consequence, the electron dose is higher without monochromator than with monochromator. Therefore, the precision is higher in the absence of a monochromator.

\section{Accelerating Voltage}

Another important problem is that at the high accelerating voltages used for HREM (200-300 keV), the amorphous structure is continuously damaged by the electron beam. It is believed that much lower voltages are needed for reliable quantitative studies. However, lowering the voltage also deteriorates the instrumental resolution. To keep the resolution at that voltage close to the intrinsic object resolution of $0.1 \mathrm{~nm}$, one will need to correct both spherical and chromatic aberration by using a $C_{s}$ corrector and either a monochromator or a $C_{c}$ corrector. As shown in Figure 2, these corrections improve the precision as well. The lower bound $s$ on the standard deviation of the position of a single projected atom is evaluated as a function of $C_{s}$ for a $50-\mathrm{keV}$ electron microscope. As in Figure 1, this has been done for the three cases discussed before. At each setting, the recording time is kept constant. From Figure 2, it is clear that the precision is highest, that is, the standard deviation is lowest, when using both a $C_{s}$ corrector and a $C_{c}$ corrector. The $C_{c}$ corrector obtains better results than the monochromator because of the higher electron dose. Moreover, this figure shows that, in the absence of a monochromator or a $C_{c}$ corrector, the precision decreases if $C_{s}$ is corrected. More results of this research are published in Van Dyck et al. (2003).

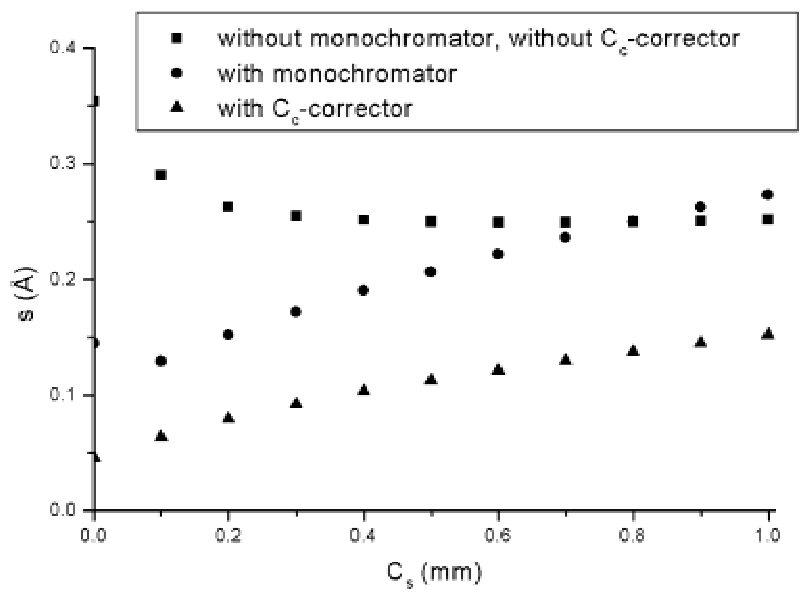

Figure 2. Lower bound $s$ on standard deviation of a single $\mathrm{Si}$ atom position as a function of $C_{s}$ for a microscope with an accelerating voltage of $50 \mathrm{keV}$. Three possible cases are distinguished: without monochromator and without $C_{c}$ corrector, with monochromator, and with $C_{c}$ corrector. At each setting, the recording time is kept constant.

\section{CONCLUSIONS}

- A fundamental limit of resolution is given by the intrinsic "width" of the atoms. For amorphous objects, this limit is reached at $300 \mathrm{keV}$, without $C_{s}$ corrector.

- Quantitative structure determination of realistic amorphous objects is impossible from one orientation, but requires electron tomography.

- The study of amorphous objects should be done at low accelerating voltage and requires correction of both spherical and chromatic aberration. A $C_{c}$ corrector is preferred rather than a monochromator for the correction of chromatic aberration.

- The precision with which atom positions can be determined is a function of both resolution and dose.

\section{RefERENCES}

Den Dekker, A.J. \& van den Bos, A. (1997). Resolution: A survey. J Opt Soc Am A 14, 547-557.

Rose, A. (1948). Television pickup tubes and the problem of vision. In Advances in Electronics and Electron Physics, Marton, L. (Ed.), pp. 131-166. New York: Academic Press.

van den Bos, A. \& den Dekker, A.J. (2001). Resolution reconsidered-Conventional approaches and an alternative. $A d v$ Imag Electron Phys 117, 241-360.

Van Dyck, D. \& Op De Beeck, M. (1996). A simple intuitive theory for electron diffraction. Ultramicroscopy 64, 99-107.

Van Dyck, D., Van Aert, S., den Dekker, A.J. \& van den Bos, A. (2003). Is atomic resolution transmission electron microscopy able to resolve and refine amorphous structures? Ultramicroscopy $98,27-42$. 